\title{
随机限食和重喂食小鼠能量收支和生长发育的可塑性
}

\author{
赵志军 ${ }^{1,2, *}$, 王瑞瑞 ${ }^{1}$, 曹 静 ${ }^{1}$, 裴兰英 ${ }^{1}$ \\ (1. 聊城大学农学院, 山东 聊城 252059; 2. 山东省生态学与生物多样性重点实验室, 山东 聊城 252059)
}

摘要: 为阐明能量收支和生长发育的可塑性对动物适应食物资源变化的作用和意义, 将断乳后的雄性 KM 小 鼠 40 只随机限食 4 周, 再重喂食 4 周。采用封闭式流体压力呼吸计测定基础代谢率(BMR)。限食使摄食量显著 增加, BMR 和活动行为降低, 胴体和生殖腺重量显著降低。重喂食后上述指标均恢复到对照组水平, 表现出显 著的可塑性变化。结果表明, KM 小鼠能通过摄食量、BMR、活动行为和身体组成的可塑性调节以适应难以预测 的食物资源变化。

关键词: 限食; 能量收支; 行为; 生长发育; 小鼠

中图分类号: Q493.8; Q494 文献标识码: A 文章编号: 0254-5853-(2009)05-0534-05

\section{Effect of Random Food Deprivation and Refeeding on Energy Budget and Development in Mice}

\author{
ZHAO Zhi-jun ${ }^{1,2,{ }^{*}}$, WANG Rui-rui ${ }^{1}$, CAO Jing ${ }^{1}$, PEI Lan-ying ${ }^{1}$ \\ (1. School of Agricultural Science, Liaocheng University, Liaocheng 252059, China; \\ 2. Shandong Key Laboratory for Ecology and Biodiversity, Liaocheng 252059, China)
}

\begin{abstract}
Significance of plasticity in energy budget and development of animals in response to variations of food availability was examined in weaned male KM mice that were acclimated to random food deprivation (FD) for 4 weeks, followed by feeding ad libtum for a following 4 weeks. Food intake was determined using a food balance method. Basal metabolic rate (BMR) was measured using a closed-circuit respirometer. Food intake increased significantly on ad libitum day in FD mice, but BMR and activity decreased. FD mice also had a significantly lower weight in carcass and gonadal gland than controls after 4 weeks of FD. All the above parameters recovered to the levels of controls after 4 weeks of ad libitum refeeding, indicating a significant plasticity. In addition, the group difference in fat content was not significant. These results suggest that animals can compensate for unpredictable lower food availability by an energetic strategy, including an increase in food intake and a decrease in energy expenditure associated with BMR and activity, and decrease in carcass mass thus reduced energy spent on maintenance, but not including the changes in body fat. Development is affected significantly by lower food availability but recovers to the normal level when food is plentiful. Finally, plasticity in the energetic budget and development play important roles in animals under unpredictable variations of food availability.
\end{abstract}

Key words: Food deprivation; Energy budget; Behavior; Development; Mice

表型可塑性(phenotypic plasticity)是指同一个 基因型对不同环境应答而产生不同表型的特性。其 中 “表型” 是和 “基因型” 相对的遗传学概念, 包 括除基因之外的所有性状, 涉及形态解剖、生理生 化以及生活史等各个方面(Vezina \& Williams, 2003; Geng et al, 2004)。表型可塑性能增强动物对各种环
境的适应性，对提高其生存能力具有重要意义 (Vezina \& Williams, 2003)。食物资源限制是动物面 临的主要应激环境之一。能量摄入和支出的权衡是 动物适应环境变化的主要策略。但在许多条件下, 能量摄入受动物消化和吸收能力的制约, 故此能量 支出在能量权衡策略中发挥的作用可能更为重要 
(Hambly \& Speakman, 2005; Zhao \& Wang, 2007; Sucajtys-Szulc et al, 2008)。基础代谢率(BMR)是动 物最主要的能量支出之一, 受食物资源的影响, 如 限食 20\%的 MF1 小鼠的 BMR 显著降低, BMR 对 适应限食应激环境的贡献率达到了 22\%(Hambly \& Speakman, 2005)。但动物是否通过 BMR 的可塑性 以适应难以预测的食物资源变化, 尚不清楚。此外, 许多动物通过调节行为活动以适应环境条件的变 化(Alvarenga et al, 2005), 如限食使 MF1 小鼠行为 活动显著降低, 但 Boakes \& Dwyer (1997)却发现限 食使大鼠的转轮行为显著增加, Wood \& Bartness (1996)也发现食物资源缺乏显著增加了黑线毛足鼠 (Phodopus sungorus)的储食行为。由此可见, 动物 的行为活动对食物资源变化的反应并不一致, 可能 具有种属差异。行为活动的调节在动物适应难以预 测的食物资源变化过程中的作用还不明确。

通过测定随机限食和重喂食 $\mathrm{KM}$ 小鼠的摄食 量、BMR、行为活动、身体组成和性腺重量, 我们 探讨在生长发育过程中动物适应食物资源变化的 能量学策略, 旨在阐明能量摄入、支出和生长发育 的可塑性在小鼠适应难以预测的食物资源变化中 所起的作用和生物学意义。

\section{1 材料和方法}

\section{1 实验动物}

$\mathrm{KM}$ 小鼠购自山东大学实验动物中心(生产许 可证号：SCXK(鲁)2003-0004)。饲养条件为：(23 $\pm 1)^{\circ} \mathrm{C}$, 光照: $12 \mathrm{~L}: 12 \mathrm{D}$, 自由取食(北京科澳协力 饲料有限公司生产的标准小鼠饲料)和饮水。经交配 繁殖后, 幼体于 19 日龄断乳, 一周后, 取 40 只雄 性小鼠单笼饲养 $(29 \mathrm{~cm} \times 18 \mathrm{~cm} \times 16 \mathrm{~cm})$, 随机分为 2 组: 对照组(Con, $n=20)$, 自由取食和饮水; 实验组 (Exp, $n=20)$, 每周随机限食 3d, 其余 $4 \mathrm{~d}$ 自由取食; 4 周后，每组随机选取 10 只测定 BMR、消化率、 行为活动以及身体成分。其余小鼠恢复自由取食, 重喂食 4 周后再测定上述指标。在限食和重喂食期 间, 每天测定摄食量, 每 3 天测定一次体重。

\subsection{BMR}

以封闭式流体压力呼吸计测定代谢率。BMR 测定温度为 $30 \pm 1^{\circ} \mathrm{C}$ (热中性区温度, Speakman \& Rossi, 1999)。测定前动物饥饿 $4 \mathrm{~h}$, 放入呼吸室进行 适应 $1 \mathrm{~h}$, 待动物稳定后开始记录, 每隔 5 min 记 录 1 次, 连续测定 $60 \mathrm{~min}$ 。选取 2 个连续稳定的最
低值计算 BMR (Wang et al, 2000; Zhao et al, 2008)。

\section{3 行为活动}

按照 Speakman et al (2001)叙述的方法将行为 活动划分为 4 类: (1)一般活动行为(general activity), 包括动物走动、奔跑、跳跃以及攀爬行为; (2)修饰 行为 (grooming behavior), 指动物的自我修饰, 如称 足、梳理皮毛等; (3)摄食行为(feeding behavior), 指取食和饮水; (4)休息行为 (resting behavior), 指动 物的任何非活动行为, 包括睡眠。每只动物观察 30 $\mathrm{s}$, 记录其优势行为活动, $10 \mathrm{~min}$ 后再按固定顺序 进行下一轮的观察，合计观察 6 次/只/h。在 06:00 -10:00 和 18:00-22:00 进行观察, 合计观察 $8 \mathrm{~h} / \mathrm{d}$, 48 次/只/天，计算每一种行为的百分率 $(\% / 8 \mathrm{~h})$ 。上 述行为活动分别在限食驯化期间的限食日(第 $26 \mathrm{~d}$ ) 和自由取食日(第 $27 \mathrm{~d})$, 以及重喂食 4 周后进行观 察。黑暗期间的观察以 $30 \mathrm{~W}$ 红色灯泡照明 (Speakman et al, 2001)。

\section{4 身体组成}

限食驯化 4 周后, 对照组和实验组各随机选取 10 只动物, 断颈处死。其余动物重喂食 4 周后处死。 分离性腺(精索、睪丸和附睪), 再分别分离性腺脂 肪、腹部和皮下脂肪, 三者之和占胴体的百分率作 为脂肪含量; 分离肝脏、心脏、肺、脾和肾脏, 分 别称重(精确至 0.01g) (Zhao \& Wang, 2006)。

\section{5 统计分析}

数据处理采用 SPSS 13.0 软件包。体重、摄食 量、BMR、行为活动、胴体重、脂肪重量和含量、 性腺重量, 以及内脏器官的组间差异均采用独立样 本 $t$ 检验进行分析。数据全部表示为平均值士标准 误(Mean $\pm S E), P<0.05$ 为差异显著, $P<0.01$ 为差异 极显著。

\section{2 结 果}

\section{1 体重、摄食量和 BMR}

实验开始时体重组间差异不显著 $\left(t_{18}=0.371\right.$, $P>0.05)$ 。此后, 二组动物体重均显著增加, 但实验 组增加幅度小于对照组, 第 $6 \mathrm{~d}$ 显著低于对照组(d6,

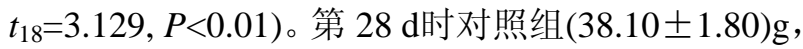
实验组 $(23.79 \pm 0.72) g$, 实验组比对照组低 $37.6 \%$ $\left(t_{18}=7.397, P<0.01\right)$ 。重喂食后实验组体重显著增加, 但 R3 和R6 d 仍显著低于对照组(R3, $t_{18}=3.658$, $P<0.01$; R6, $\left.t_{18}=2.224, P<0.05\right)$, 但自重喂食第 $9 \mathrm{~d}$, 组间差异不显著 $\left(\mathrm{R} 28, t_{18}=0.555, P>0.05\right.$, 图 $\left.1 \mathrm{~A}\right)$ 。 
实验开始时摄食量组间差异不显著 $\left(t_{18}=0.051\right.$, $P>0.05)$ 。在随机限食期间, 实验组分别在第 5、7、 11、14 28 d 显著高于对照组。第 $28 \mathrm{~d}$, 对照组为 $(5.59 \pm 0.31) \mathrm{g} / \mathrm{d}$, 实验组为 $(11.18 \pm 0.24) \mathrm{g} / \mathrm{d}$, 后者 比前者高 $99.8 \%\left(t_{18}=14.349, P<0.001\right)$ 。重喂食后实 验组摄食量显著降低, 但R1 R4 d仍显著高于对照 组( $\left.R 4, t_{18}=2.333, P<0.05\right)$ 。但自重喂食第 $5 \mathrm{~d}$ 后, 组 间差异不显著 $\left(R 28, t_{18}=1.472, P>0.05\right.$, 图 1B)。限食 4 周使动物整体 $\mathrm{BMR}\left(\mathrm{mlO}_{2} / \mathrm{h}\right)$ 显著降低, 但单位体 重BMR $\left(\mathrm{mlO}_{2} / \mathrm{g} / \mathrm{h}\right)$ 组间差异不显著。重喂食 4 周后, BMR 组间差异不显著(表 1)。

\section{2 行为活动}

随机限食驯化 4 周, 实验组限食日的活动行为
显著高于对照组 $\left(t_{18}=5.664, P<0.01\right.$, 图 $\left.2 \mathrm{~A}\right)$, 而修饰 行为显著低于对照组 $\left(t_{18}=2.694, P<0.05\right)$, 休息行为 组间差异不显著 $\left(t_{18}=1.230, P>0.05\right)$ 。实验组自由取 食日的活动和修饰行为均显著低于对照组(活动, $t_{18}=2.667, P<0.05$; 修饰行为, $t_{18}=3.008, P<0.05$, 图 $2 B)$, 但取食行为显著高于对照组 $\left(t_{18}=3.767\right.$, $P<0.01$ )。重喂食后, 上述行为组间差异均不显著(活 动, $t_{18}=1.594, P>0.05$; 取食行为, $t_{18}=1.863, P>0.05$; 修饰行为, $t_{18}=2.001, P>0.05$; 休息行为, $t_{18}=2.038$, $P>0.05$; 图 2C)。

\section{3 身体组成}

随机限食 4 周使胴体、性腺脂肪、心脏、肾 脏, 以及精索和睪丸的重量均显著降低, 但对腹部

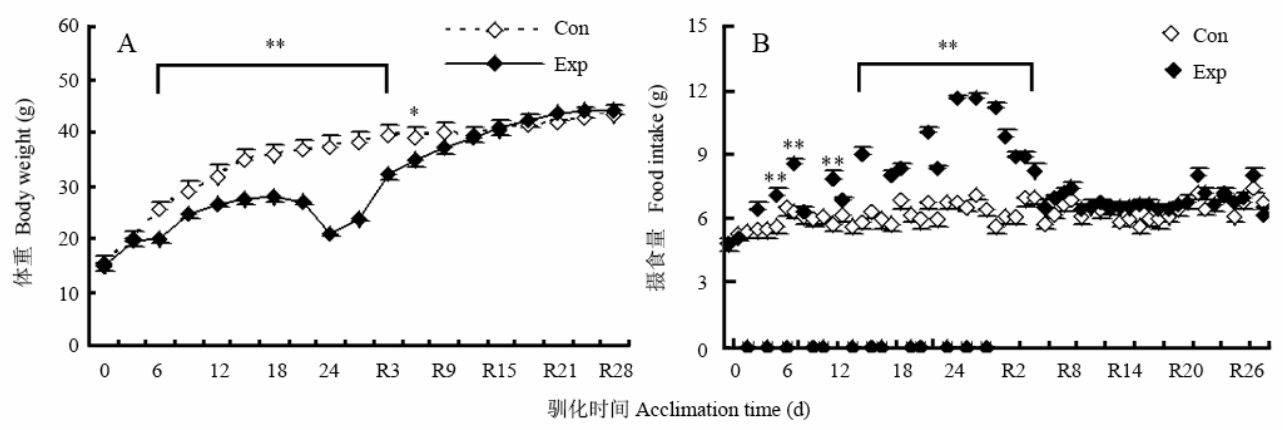

图 1 随机限食和重喂食对雄性 $\mathrm{KM}$ 小鼠体重和摄食量的影响

Fig. 1 Effect of random food deprivation and refeeding on body weight and food intake in male KM mice Con: 对照组; Exp：随机饥饿 4 周再重喂食 4 周组。数值为平均值士标准误。* $P<0.05 ; * * P<0.01$ 。

Con: control individuals, Exp: the animals that were stochastically deprived of food for 4 weeks and then refed ad libitum for another 4 weeks. Values are presented as mean $\pm S E$. $* P<0.05 ; * * P<0.01$.
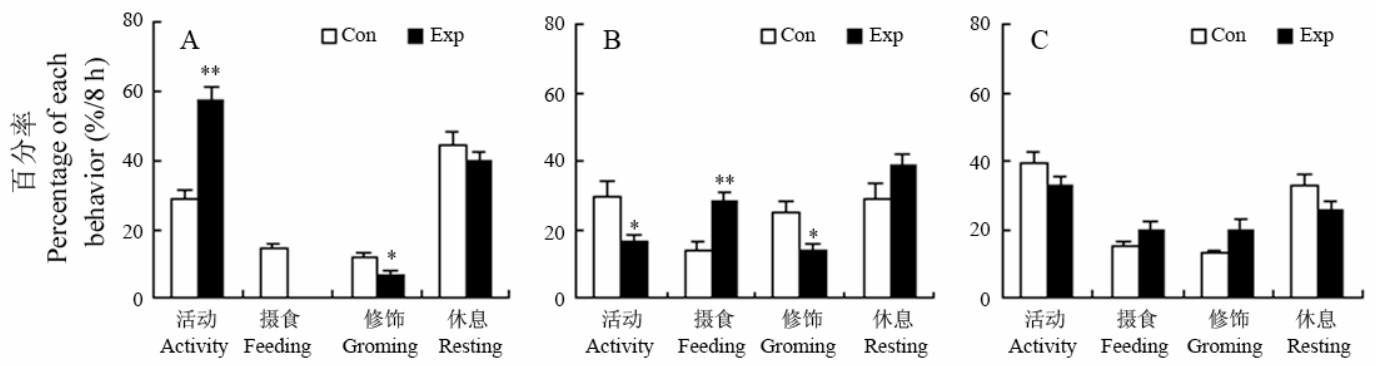

图 2 随机限食和重喂食对雄性 $\mathrm{KM}$ 小鼠行为活动的影响

Fig. 2 Effect of random food deprivation and refeeding on behavioral patterns in male KM mice $\mathrm{A}, \mathrm{B}$ 分别为限食期间限食日和自由取食日的行为; C 为重喂食期间的行为; Con：对照组; Exp：随机饥饿 4 周再重喂食 4 周组。数值为平 均值士标准误。* $P<0.05$; ** $P<0.01$ 。

A and B, behavior on deprived day and ad libitum day in response to random food deprivation; C, behavior on ad libitum day during refeeding; Con, control individuals, Exp, the animals that were stochastically deprived of food for 4 weeks and then refed ad libitum for another 4 weeks. Values are presented as mean $\pm S E$. $* P<0.05,{ }^{* *} P<0.01$.

脂肪、皮下脂肪、脂肪含量, 以及附睪的重量的影 响均未达到显著水平。重喂食 4 周后上述各参数的 组间差异均不显著(表 1)。

\section{3 讨 论}

随机限食和重喂食显著影响 KM 小鼠体重、摄 
食量、身体组成和行为活动。随机限食使摄食量增 加, 活动行为和 BMR 降低, 胴体和性腺重量均显
著降低。重喂食后上述生理指标均恢复至对照组水 平，表现出显著的可塑性。

表 1 随机限食和重喂食对小鼠 BMR 和身体组成的影响

Tab. 1 Effect of random food restriction and recovery on BMR and body composition in mice

\begin{tabular}{|c|c|c|c|c|c|c|c|c|}
\hline & $\begin{array}{l}4 \text { 周对照组 } \\
4 \text { w control }\end{array}$ & $\begin{array}{l}\text { 随机限食组 } \\
\text { FD }\end{array}$ & $T$ & $P$ & $\begin{array}{l}8 \text { 周对照组 } \\
8 \text { w control }\end{array}$ & $\begin{array}{c}\text { 限食+重喂食 } \\
\text { 组 FR-Re }\end{array}$ & $T$ & $P$ \\
\hline $\begin{array}{l}\text { 体重 Body mass (g) } \\
\text { 基础代谢率 BMR }\end{array}$ & $40.50 \pm 1.25$ & $27.58 \pm 1.50$ & 6.78 & $* *$ & $45.83 \pm 1.73$ & $48.20 \pm 0.72$ & 1.19 & ns \\
\hline $\mathrm{mlO}_{2} / \mathrm{h}$ & $2.46 \pm 0.10$ & $2.61 \pm 0.16$ & 0.78 & ns & $2.32 \pm 0.15$ & $2.63 \pm 0.12$ & 1.58 & ns \\
\hline $\mathrm{mlO}_{2} / \mathrm{h} / \mathrm{g}$ & $93.64 \pm 4.20$ & $66.90 \pm 5.38$ & 3.92 & $* *$ & $98.42 \pm 7.26$ & $113.01 \pm 6.61$ & 1.49 & ns \\
\hline 胴体 Carcass mass (g) & $27.40 \pm 0.78$ & $18.39 \pm 0.91$ & 7.50 & $* *$ & $30.50 \pm 0.96$ & $31.41 \pm 1.02$ & 0.65 & ns \\
\hline $\begin{array}{l}\text { 腹部脂肪 Abdominal fat } \\
\text { (g) }\end{array}$ & $0.90 \pm 0.07$ & $0.75 \pm 0.09$ & 1.30 & ns & $1.33 \pm 0.14$ & $1.55 \pm 0.14$ & 1.10 & ns \\
\hline $\begin{array}{l}\text { 皮下脂肪 Subcutaneous } \\
\text { fat (g) }\end{array}$ & $0.63 \pm 0.08$ & $0.70 \pm 0.20$ & 0.32 & ns & $0.82 \pm 0.20$ & $1.23 \pm 0.26$ & 1.24 & ns \\
\hline 性腺脂肪 Gonad fat (g) & $0.54 \pm 0.05$ & $0.21 \pm 0.06$ & 4.30 & $* *$ & $0.79 \pm 0.14$ & $1.05 \pm 0.13$ & 1.33 & ns \\
\hline $\begin{array}{l}\text { 脂肪含量 Body fat content } \\
(\%)\end{array}$ & $7.54 \pm 0.51$ & $8.79 \pm 1.49$ & 0.79 & ns & $9.51 \pm 1.22$ & $12.09 \pm 1.48$ & 1.36 & ns \\
\hline 内脏器官 Organ mass (g) & & & & & & & & \\
\hline 肝 Liver & $2.04 \pm 0.11$ & $2.55 \pm 0.13$ & 2.93 & * & $2.50 \pm 0.09$ & $2.88 \pm 0.09$ & 2.94 & $*$ \\
\hline 心 Heart & $0.18 \pm 0.01$ & $0.16 \pm 0.01$ & 2.51 & * & $0.21 \pm 0.01$ & $0.22 \pm 0.01$ & 1.23 & ns \\
\hline 肺 Lung & $0.22 \pm 0.01$ & $0.181 \pm 0.01$ & 2.18 & ns & $0.22 \pm 0.01$ & $0.25 \pm 0.01$ & 2.49 & $*$ \\
\hline 脾 Spleen & $0.12 \pm 0.01$ & $0.09 \pm 0.01$ & 2.17 & ns & $0.10 \pm 0.01$ & $0.12 \pm 0.02$ & 1.10 & ns \\
\hline 肾 Kidneys & $0.59 \pm 0.04$ & $0.47 \pm 0.03$ & 2.35 & * & $0.67 \pm 0.04$ & $0.68 \pm 0.02$ & 0.23 & ns \\
\hline 生殖腺 Gonad (g) & & & & & & & & \\
\hline 精索 Varicosity & $0.35 \pm 0.01$ & $0.12 \pm 0.02$ & 10.35 & $* *$ & $0.34 \pm 0.03$ & $0.34 \pm 0.03$ & 0.14 & ns \\
\hline 睪丸 Testis & $0.28 \pm 0.02$ & $0.23 \pm 0.01$ & 3.11 & * & $0.27 \pm 0.02$ & $0.29 \pm 0.02$ & 1.01 & ns \\
\hline 附睪 Epididymis & $0.05 \pm 0.01$ & $0.04 \pm 0.01$ & 1.72 & ns & $0.06 \pm 0.01$ & $0.06 \pm 0.01$ & 0.53 & ns \\
\hline
\end{tabular}

\section{1 体 重}

许多小型哺乳动物的体重受食物质量或者食 物资源的影响(Alvarenga et al, 2005; Zhao \& Wang, 2007; Zhang \& Wang, 2008)。我们研究发现, 随机 限食使雄性 KM 小鼠体重显著低于对照组, 生长发 育受到抑制。与我们的研究结果相似, 限食 $25 \%$ 和 $50 \%$ 使大鼠体重分别降低了 $22 \%$ 和 $40 \%$ (Sucajtys-Szulc et al, 2008)。但也有研究发现限食 $20 \%$ 小鼠和 $30 \%$ 大鼠的体重未出现显著变化 (Hambly \& Speakman, 2005)。这些研究结果似乎表 明, 动物体重的变化受限食程度的影响。若限食幅 度较小, 动物能通过提高消化道的吸收效率增加能 量摄入, 调整代谢率和日常行为活动调节能量支 出。然而当限食程度增加, 动物可能无法通过上述 调节途径以弥补能量摄入的不足, 从而表现为生长 发育缓慢, 体重降低(Alvarenga et al, 2005; Zhang \& Wang, 2008)。重喂食后, KM 小鼠体重迅速恢复到 了对照组水平, 表现出了显著的可塑性调节。体重 的可塑性取决于能量摄入和能量支出的变化。

\section{2 食物摄入和代谢能量支出}

随机限食期间 KM 小鼠的摄食量在自由取食日 显著增加, 重喂食后降低, 逐渐恢复到了对照组水 平。随机限食使 $\mathrm{KM}$ 小鼠 BMR 显著降低。限食也 使大鼠、小鼠和狝猴的代谢率降低(McCarter \& Palmer, 1992; DeLaney et al, 1999)。 Hambly \& Speakman (2005)计算了限食 20\%的 MF1 小鼠的 RMR, 认为 RMR 在适应限食的补偿策略中所占的 贡献为 22\% (Hambly \& Speakman, 2005)。重喂食后 摄食量和 BMR 均恢复到了对照组水平, 亦表现出 显著的可塑性调节。BMR 是小型哺乳动物最主要 的能量支出之一, 在能量平衡的调节中发挥重要作 用。许多动物通过改变 BMR 调节能量代谢, 以适 应环境条件的变化 (Hambly \& Speakman, 2005; Zhao \& Wang, 2007; Zhang \& Wang, 2008)。上述研 究表明, 在食物资源发生难以预测的变化时, 动物 不仅通过改变摄食量调节能量摄入, 而且通过基础 代谢率的可塑性变化, 调节能量支出。限食和重喂 食对脂肪含量的影响未达到显著水平，暗示并未动 用身体它存的能量。而随机限食使胴体显著低于对 照组, 表明 KM 小鼠通过降低自身维持所需的能量 
以降低能量支出也是应对随机限食的能量学策略 之一。

\section{3 行 为}

动物通常改变它们的行为活动以适应环境的 变化。我们发现, 随机限食使限食日 $\mathrm{KM}$ 小鼠的活 动行为增加, 而自由取食日活动降低。限食亦导致 大鼠的转轮活动显著增加(Sherwin, 1998)。这些结 果可能暗示, 当食物资源限制或者不可预测时, 动 物增加活动行为, 寻受食物(Sherwin, 1998; Hambly \& Speakman, 2005)。但获得食物期间，KM 小鼠的 活动行为显著降低。限食也使小鼠的活动行为减少 (Hambly \& Speakman, 2005), 使大鼠的睡眠行为增 加(Alvarenga et al, 2005), 表明动物可能通过减少活 动行为, 降低能量支出, 以补偿短缺的食物资源。 重喂食后, 活动行为、修饰、取食和休息行为均恢 复到了对照组水平, 亦表现出显著的可塑性调节。 这些研究结果说明, 在随机限食条件下 KM 小鼠不

\section{参考文献:}

Alvarenga TAF, Andersen ML, Papale LA, Antunes IB, Tufik S. 2005. Influence of long-term food restriction on sleep pattern in male rats [J]. Brain Res, 1057: 49-56.

Boakes RA, Dwyer DM. 1997. Weight loss in rats produced by running: Effects of prior experience and individual housing [J]. J Exp Psychol B50: $129-48$.

DeLaney JP, Hansen BC, Bodkin DL, Hannah J, Bray G.A. 1999. Long-term caloric restriction reduces energy expenditure in aging monkeys [J]. $J$ Gerontol Biol Sci, 54A: 5-11.

Geng YP, Zhang WJ, LI B, Cheng JK. 2004. Phenotypic plasticity and invasiveness of alien plants [J]. Biodiver Sci, 12(4): 447-455. [耿宇鹏, 张文驹, 李 博, 陈家宽. 2004. 表型可塑性与外来植物的入侵能 力. 生物多样性, 12(4): 447-455.]

Hambly C, Speakman JR. 2005. Contribution of different mechanisms to compensation for energy restriction in the mouse [J]. Obes Res, 13: 1548-1557.

McCarter RJM, Palmer J. 1992. Energy metabolism and aging: a lifelong study in Fischer 344 rats [J]. Am J Physiol, 263ED: 448-452.

Sherwin CM. 1998. Voluntary wheel running: a review and novel interpretation [J]. Anim Behav, 56: 11-27.

Speakman JR, Gidney A, Bett J, Mitchell IP, Johnson MS. 2001. Limits to sustained energy intake IV. Effect of variation in food quality on lactating mice Mus Musculus [J]. J Exp Biol, 204: 1957-1965.

Speakman JR, Rossi F. 1999. No support for socio-physiological suppression effect on metabolism of paired white mice (Mus sp.) [J]. Funct Ecol, 13: 373-382.

Sucajtys-Szulc E, Goyke E, Korczynska J, Stelmanska E, Rutkowski B,
仅通过增加自由取食期间的摄食量以增加能量摄 入, 而且通过降低活动行为, 减少能量支出。行为 活动的生理可塑性变化, 对于动物适应难以预测的 食物资源短缺环境同样具有重要意义。

总之，与正常发育的动物相比，随机限食使 KM 小鼠体重、BMR、胴体和性腺重量均显著降低, 摄食量增加。随机限食还会导致自由取食日的活动 行为降低, 限食日的活动行为增加。重喂食后, 上 述指标均恢复到对照组水平，表现出显著的可塑性 变化。但随机限食和重喂食未显著影响脂肪含量。 这些研究结果表明，小鼠适应难以预测的食物资源 短缺的策略包括：增加摄食量以增加能量摄入; 降 低 BMR 和活动行为以减少能量支出, 降低胴体重 以减少自身维持的所需的能量, 而非动员机体的能 量贮存。能量摄入、支出和生长发育的可塑性对小 鼠适应难以预测的食物资源变化的环境具有重要 作用和意义。

Swierczynski J. 2008. Chronic food restriction differentially affects NPY mRNA level in neurons of the hypothalamus and in neurons that innervate liver [J]. Neurosci Lett, 433: 174-177.

Vezina F, Williams TD. 2003. Plasticity in Body Composition in Breeding Birds: What Drives the Metabolic Costs of Egg Production? [J]. Physiol Biochem Zool, 76: 716-730.

Wang DH, Wang YS, Wang ZW. 2000. Metabolism and thermoregulation in the Mongolian gerbil (Meriones unguiculatus) [J]. Acta Theriol Sin, 45: 183-192.

Wood AD, Bartness TJ. 1996. Food deprivation-induced increases in hoarding by Siberian hamsters are not photoperiod-dependent [J]. Physiol Behav, 60: 1137-1145.

Zhang LN, Wang DH. 2008. Effects of food restriction and refeeding on energy balance regulation in Mongolian gerbils (Meriones unguiculatus) [J]. BFDG Abstracts/Appetite, 51: 751-764.

Zhao ZJ, Cheng JF, Wang DH. 2008. Effects of photoperiod and high fat diet on energy intake and thermogenesis in Brandt' svoles Lasiopodomysbrandtii [J]. Acta Zool Sin, 54 (4): 576-589. [赵志军, 陈 竞峰, 王德华. 2008. 光周期和高脂食物对布氏田鼠能量代谢和产 热的影响. 动物学报, 54 (4): 576-589.]

Zhao ZJ, Wang DH. 2006. Effect of photoperiod on body mass, energy balance and thermogenesis in Mongolian gerbils [J]. J Therm Biol, 31: 323-331.

Zhao ZJ, Wang DH. 2007. Effects of diet quality on energy budgets and thermogenesis in Brandt's voles [J]. Comp Biochem Physiol, 148A 168-177. 